\title{
MASTER \\ SELF-CONSISTENT CALCULATIONS \\ OF EDGE TEMPERATURE \\ AND SELF-SPUTTERING OF THE LIMITER SURFACE \\ FOR TOKAMAK FUSION REACTORS
}

by

deffrey Brooks

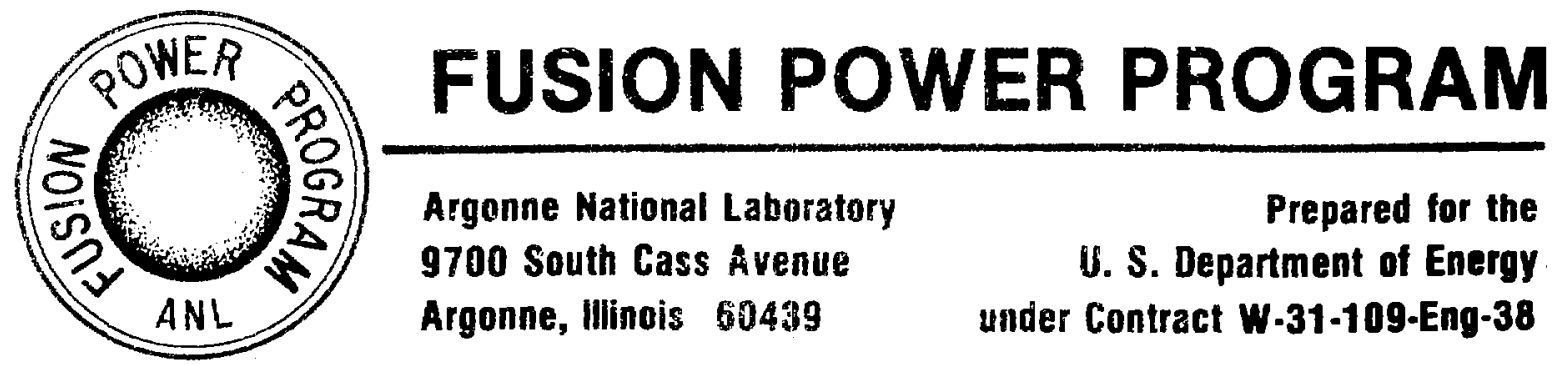


Di stribution Categories:

Magnetic Fusion Energy (UC-20)

Plasma Systems (UC-20a)

Magnetic Systems (UC-20b

Theoretical Plasma Physics (UC-20g)

ANL/FPP/TM-133

ARGONNE NATIONAL LABORATORY

9700 South Cass Avenue

Argonne, Illinois 60439

SELF-CONSISTENT CALCULATIONS OF EDGE TEMPERATURE

AND SELF-SPUTTERING OF THE LIMITER SURFACE

FOR TOKAMAK FUSION REACTORS

by

Jeffrey N. Brooks

Fusion Power Program

May 1980

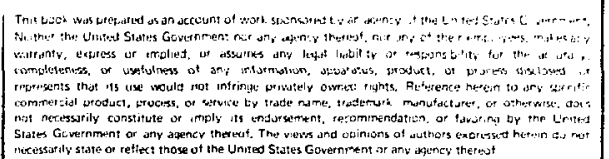




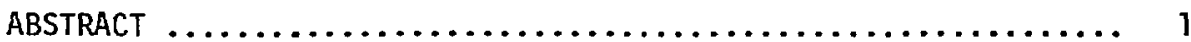

I. INTRODUCTION $\ldots \ldots \ldots \ldots \ldots \ldots \ldots \ldots \ldots \ldots \ldots \ldots \ldots \ldots \ldots \ldots \ldots \ldots, 2$

II. MODEL $\ldots \ldots \ldots \ldots \ldots \ldots \ldots \ldots \ldots \ldots \ldots \ldots \ldots \ldots \ldots \ldots \ldots \ldots \ldots \ldots \ldots \ldots \ldots, 2$

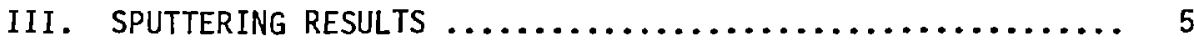

IV. EDGE-TEMPERATURE ESTIMATES $\ldots \ldots \ldots \ldots \ldots \ldots \ldots \ldots \ldots \ldots \ldots$

\section{LIST OF FIGURES}

No.

Title

Page

1 Normalized sheath potential and heat transmission factor as a function of electron re-emission coefficient

2 Self-sputtering coefficients for several low-Z coatings and structural materials

3 Self-sputtering coefficients for several low-Z compounds for $R_{e}=0$

4 Self-sputtering coefficients for several low-Z compounds for $R_{e}=0.9 \ldots \ldots \ldots \ldots \ldots \ldots \ldots \ldots \ldots \ldots \ldots \ldots \ldots \ldots \ldots \ldots \ldots \ldots \ldots \ldots, 8$

5 Edge temperature for $R_{e}=0 \ldots \ldots \ldots \ldots \ldots \ldots \ldots \ldots \ldots, 12$

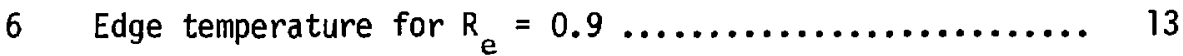




\title{
SELF-CONSISTENT CALCULATIONS OF EDGE TEMPERATURE AND SELF-SPUTTERING OF THE LIMITER SURFACE FOR TOKAMAK FUSION REACTORS
}

Jeffrey N. Brooks

Fusion Power Program

\begin{abstract}
Self-sputtering and edge temperature estimates have been made for a tokamak fusion reactor with a normal incidence boundary using models for the power balance, plasma sheath, charge state, and sputtering coefficients. Both structural materials and low- $Z$ coatings have been examined. From the self-sputtering standpoint, some materials will work only at very low edge temperatures; these will require a high recycling rate and a high radiation fraction.
\end{abstract}




\section{INTRODUCTION}

Sputtering in future tokamak reactors will depend on a number of factors all bearing on the energy of particles hitting the boundary. These factors are primarily the plasma-edge temperature, the sheath potential, and the charge state of ions entering the sheath. The choice of boundary materials will depend critically on sputtering and, in particular, on the self-sputtering coefficient of the material. The purpose of this work is to examine the likely range of sputtering coefficients and plasma-edge temperatures that might prevail for future fusion reactors. The sputtering coefficients have been calculated using models for the factors mentioned above. Edge temperature estimates have been made using a simple power balance model applied to a 3000-MW(th) tokamak reactor. The assumed boundary is that of a limiter where all of the charged particle flux from the plasma hits the limiter; the first wall would be sputtered by charge-exchange neutrals. Such a limiter and associated vacuum system have been proposed as the impurity control device of the STARFIRE [1] commercial tokamak design. The model used in this analysis applies strictly to the case of a limiter oriented perpendicular to the magnetic field but the results should reasonably apply to moderately off-normal configurations. The results should also apply to most types of divertor plates.

\section{MODEL}

The model used to compute sputtering is that, for an electron edge temperature $T_{e}$, an impurity ion in charge state $\bar{Z}=\bar{Z}\left(T_{e}\right)$ at the plasma edge flows along a magnetic field line until it gets to the vicinity of the boundary. It is then accelerated through a sheath potential $\phi$. The charge state $\bar{Z}$ is computed using the average ion model formulas of ref. 2. For the assumption of equal ion and electron edge temperatures, the impurity ion will hit the boundary at an average energy $E=3 / 2 \mathrm{KT} \mathrm{T}_{\mathrm{e}}+\mathrm{e} \bar{Z}_{\phi}$, where $3 / 2 \mathrm{KT}$ is the initial energy of the ion and $e \bar{z}_{\phi}$ is the energy acquired in the sheath. Because the energy 
acquired in the sheath is, in most cases, many times the initial energy, the energy distribution for arriving impurity ions is approximately monoenergetic. The sputtering coefficient is then computed using the model of Smith $[3,4]$ :

$$
S(E)=\frac{20}{U_{0}} Z_{1} Z_{2} \frac{M_{1}}{M_{2}} \frac{\left(E-E_{t h}\right)}{\left(E-E_{t h}+50 Z_{1} Z_{2}\right)^{2}} ;
$$

where $S$ is the sputtering coefficient in atoms per incident ion, $E$ is the incident particle energy in $a V, U_{0}$ is the surface binding energy in $e V$, and $Z$ and $M$ are the atomic and mass numbers, respectively, with the subscripts 1 and 2 referring to the incident particle and target atoms, respectively. $E_{t h}$ is the threshold energy as given in reference 4. For self-sputtering of elemental wall materials, $Z_{1}=Z_{2}$ and $M_{1}=M_{2}$. For compound wall materials or coatings, $Z_{2}$ and $M_{2}$ are interpreted as per ref. 3 .

The sheath set up at a boundary perpendicular to the magnetic field and the role of electron re-emission has been treated by various authors (e.g. see refs. 5 and 6 ). Results from an exact numerical solution of kinetic equations [7] have been employed in this work. The sheath parameters of interest are the potential $\phi$ and the heat transmission factor $\gamma$ (defined in section 4 ). These, from ref. 7, are shown in Fig. 1 as a function of the electron re-emission coefficient of the boundary material, and as a function of the ratio " $\alpha$ " of the ion-to-e lectron average kinetic energy at the start of the sheath region. These curves are for a DT plasma containing $5 \%$ beryllium and a correspondingly higher electron density than DT density, believed to be typical conditions of future devices (e.g. see ref. 1). The curves are similar if small amounts of other impurities are substituted for beryllium. The curves terminate at the so-called space charge limit at which point the electron re-emission saturates. The range of a shown varies from the Bohm limit which is the smallest value of a giving rise to a stable (time-independent) sheath to a fairly high value of $\alpha=8$. 


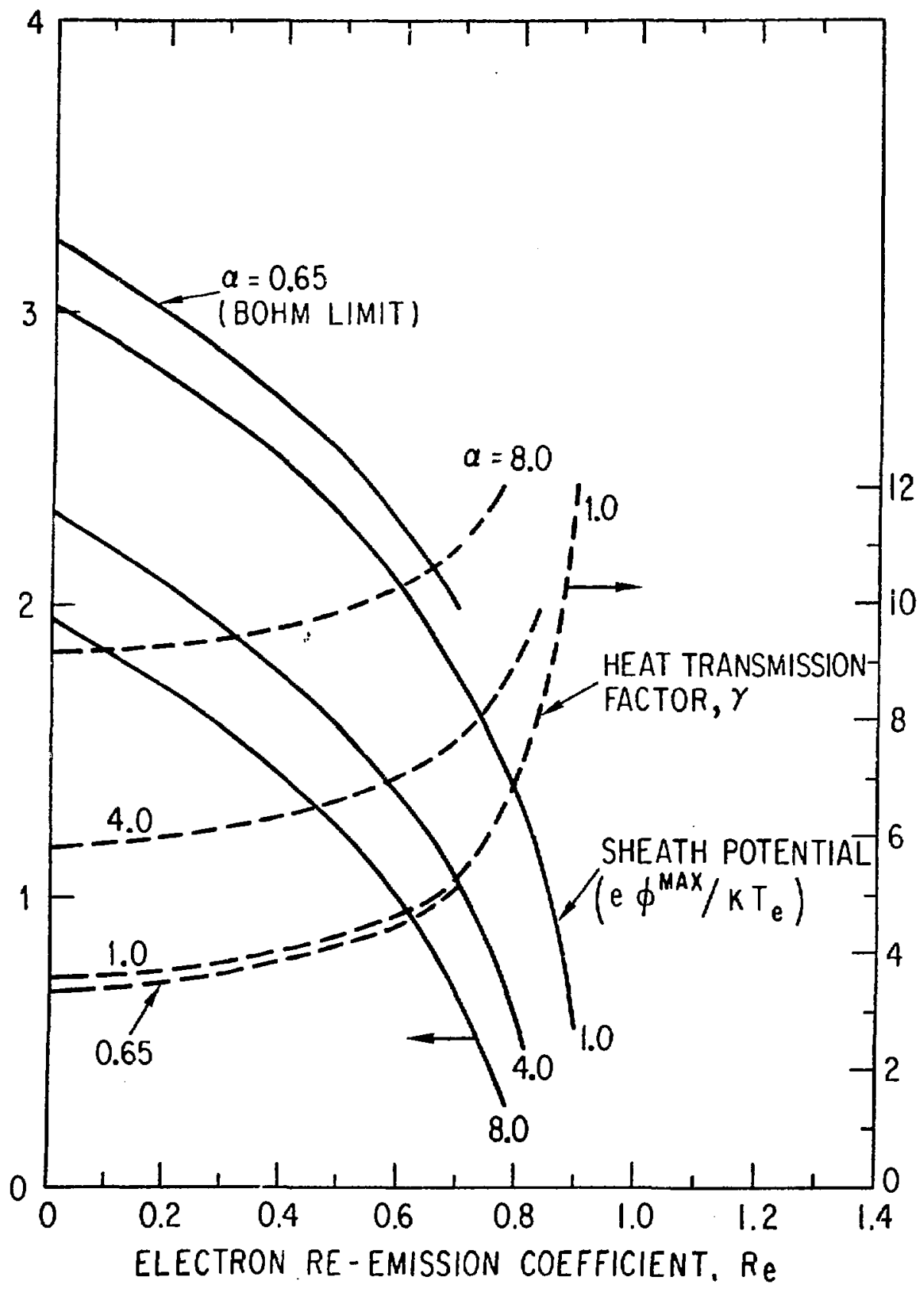

Fig. 1. Normalized sheath potential and heat transmission factor as a function of electron re-emission coefficient. From ref. 7. 
Although the value of $\alpha$ for future devices is uncertain, there are no major qualitative differences in sheath parameters with $\alpha$ (provided $\alpha$ is greater than the Bohm limit); a value of $\alpha=1$ has been used for this study.

The electron re-emission coefficient, $R_{e}$, defined as the ratio of the flux of electrons from the boundary to the flux of electrons to the boundary, is an important parameter for this analysis. As shown in Fig. 1, $\phi$ varies by a factor of about 6 to 1 and $\gamma$ by 4 to 1 over the range of $R_{e}$. The contributions to $R_{e}$ are due to electron reflection, secondary emission by electrons, and secondary emission by ions, the first two being the most important. For carbon, and metallic boundary materials, the model used to compute $R_{e}$ as a function of edge temperature was to fit a curve to the "normalized yield" data shown in Fig. 13 of ref. 8, using the parameters $\delta^{\max }$ and $E_{p}^{\max }$ as listed in Table 6-2 of ref. 9. The energy of electrons hitting the boundary is taken as their average energy. Because secondary electron data is less certain for compound materials, these have been analyzed by performing two limiting calculations, one for $R_{e}=0$ and one for $R_{e}$ at saturation $\left(R_{e} \approx 0.9\right)$.

\section{SPUTTERING RESULTS}

Figure 2 shows the results of the self-sputtering calculations for two low-Z coating materials and several candidate structural materials. Figures 3 and 4 show the results for several compound coating materials for the two limiting sheath potential cases discussed above. Also computed, but not shown for simplicity, were $V, T i, T i B_{2}$, and $T i C$. The shape of the curves is a result of the various interacting processes. For example, for iron at an edge temperature of $50 \mathrm{eV}: \bar{Z}=7.0, \mathrm{R}_{\mathrm{e}}=0.67$, e $\phi=92 \mathrm{eV}, \mathrm{E}=722 \mathrm{eV}$, and $\mathrm{S}=1.28$; at $\mathrm{T}_{\mathrm{e}}=$ $200 \mathrm{eV}, \bar{Z}=14.2, R_{e}=0.9, \mathrm{e} \phi=112 \mathrm{eV}, E=1890 \mathrm{eV}$, and $S=3.15$. The dips in some of the curves in fig. 2 occur at the point where the electron re-emission approaches saturation. The sharp reduction in sheath potential is then enough 


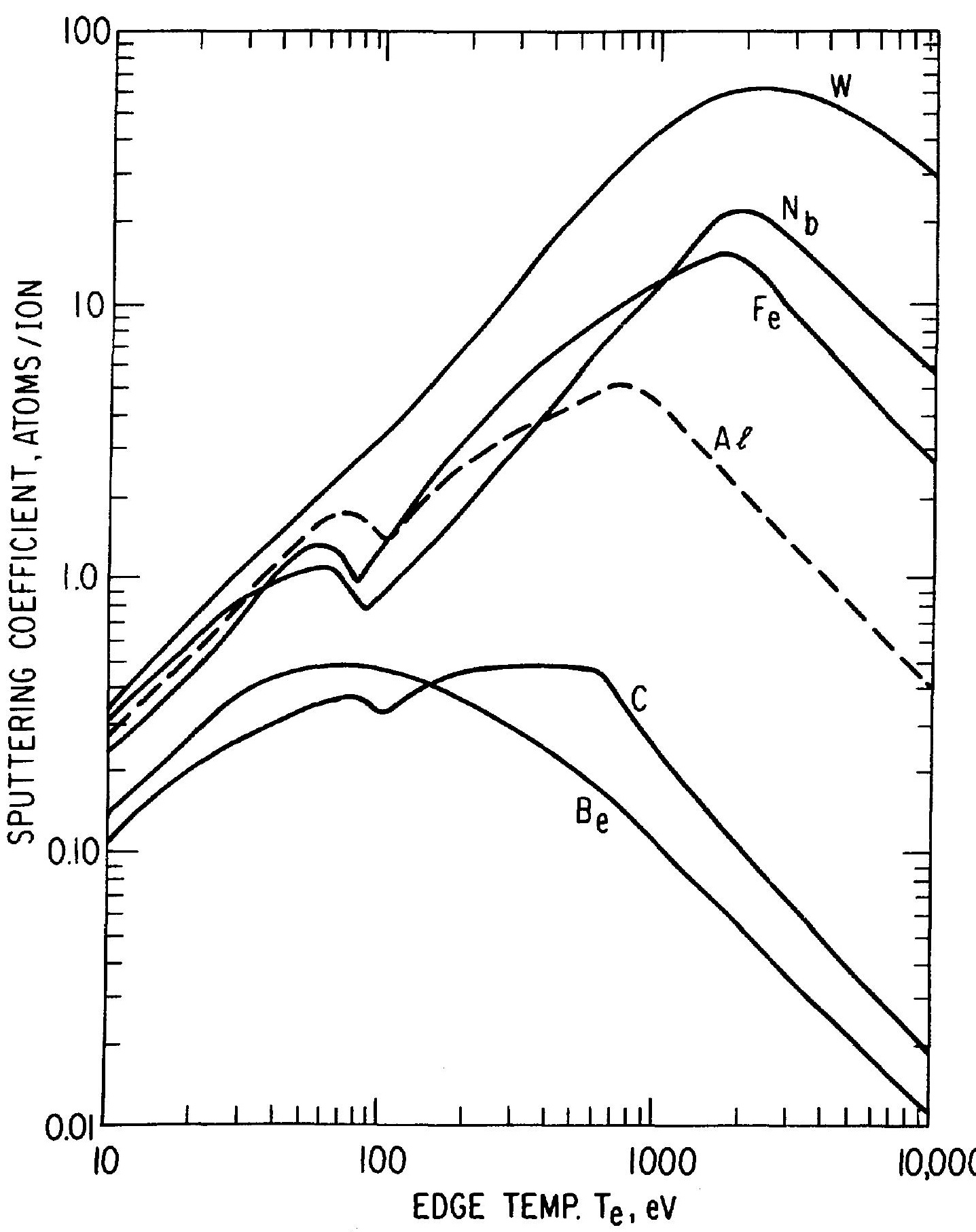

Fig. 2. Self-sputtering coefficients for several low-Z coatings and structural materials. 


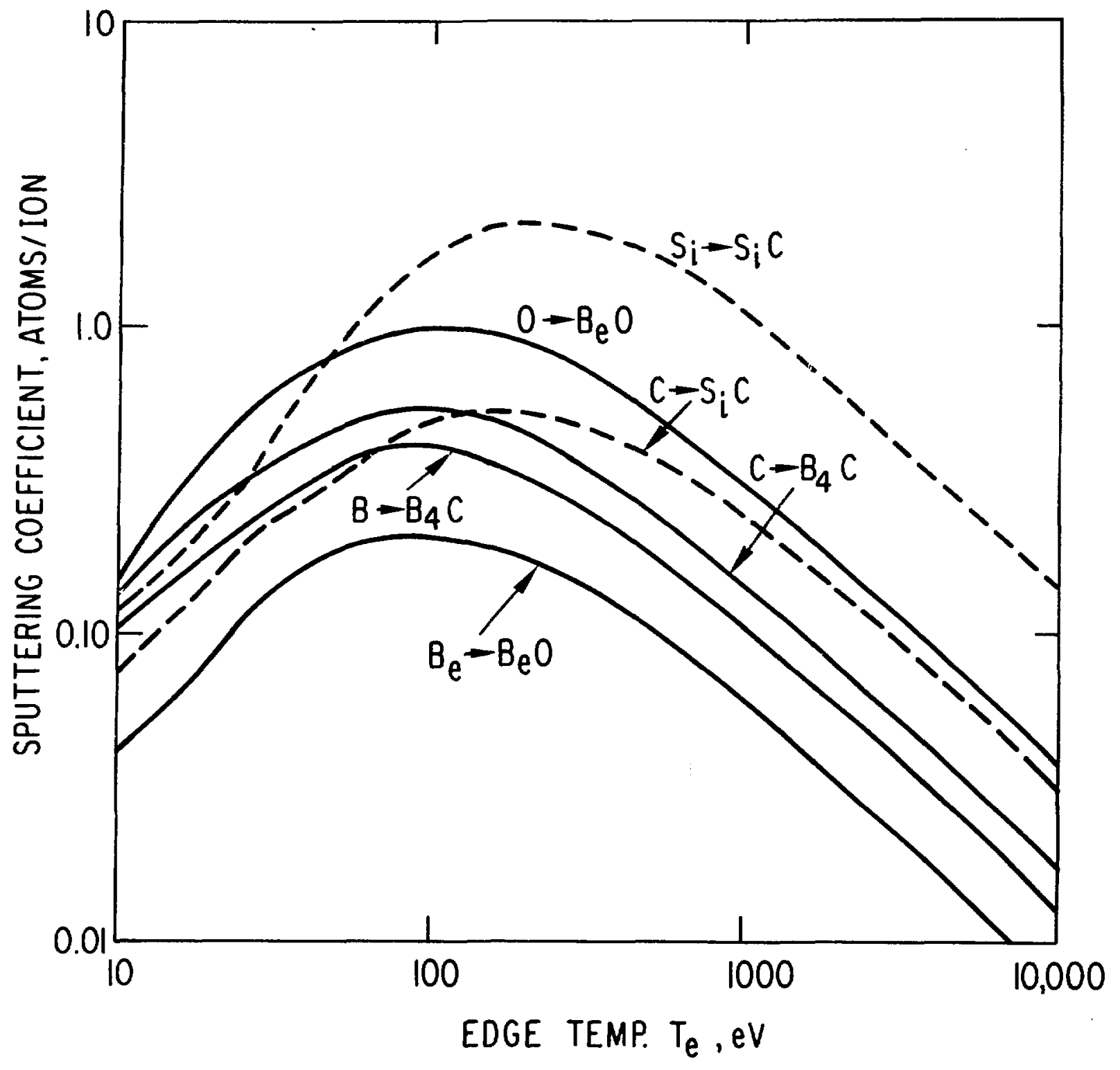

Fig. 3. Self-sputtering coefficients for several low-Z compounds for $R_{e}=0$. 


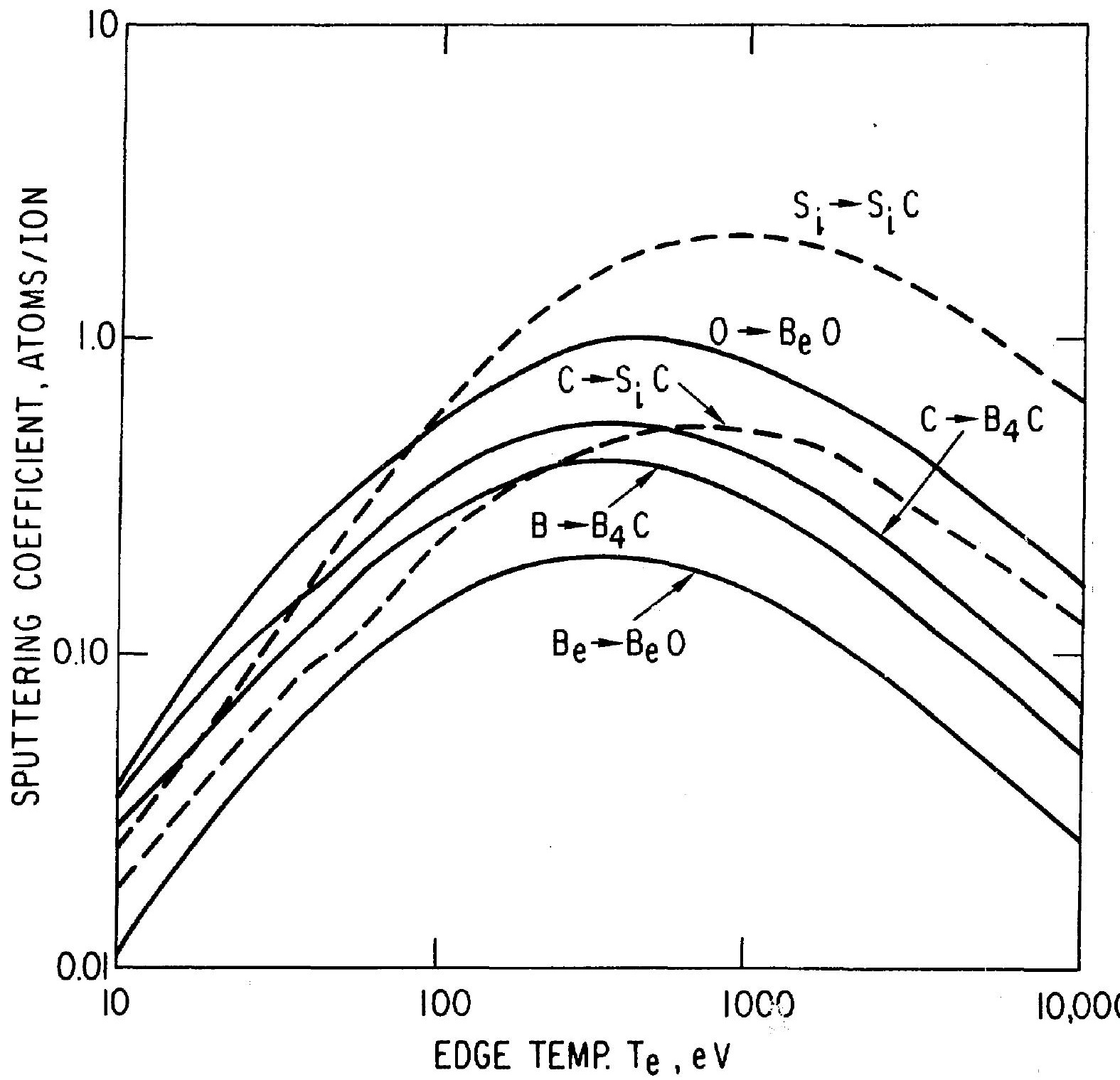

Fig. 4. Self-sputtering coefficients for several low-Z compounds for $R_{e}=0.9$. 
to temporarily offset the higher edge temperature. For a limiter impurity control system, of the type described $r$ ref. 1 , it is a necessary condition that the self-sputtering yield, for elemenu, li materials, be less than unity. If this condition is not met, then an exponentially growing sputtering cascade would result which would quickly terminate the plasma. For a divertor this condition is not quite as critical because of the divertor action; however, erosion of the divertor plate can still be a serious concern. Even if $S<1$, the magnitude of the self-sputtering coefficient is important becaus 2 the concentration of impurity in the plasma scales approximately as $N_{z} \sim(1-S)^{-1}$. As shown for the elements, beryllium and carbon always meet the criterion that $S<1$. For the higher-Z metals, this criterion is only met for $T_{e} \leqslant 40$ eV for tungsten, iron, and aluminum; and for $T_{e} \leqslant 50 \mathrm{eV}$ and $75 \leqslant \mathrm{~T}_{e} \leqslant 115 \mathrm{eV}$ for niobium. For aluminum the self-sputtering coefficient also falls below unity for $T_{e} \geq 3.5 \mathrm{keV}$. Results for vanadium and titanium are similar to those for iron. For compounds the relevant criterion is that the average self-sputtering coefficient must be less than unity where the average is taken using the fractional makeup of the compound. For binary compounds, e.g. Be0, the plasma should contain equal amounts of beryllium and oxygen, both of which should cause sputtering by virtue of equal fluxes to the boundary. The average selfsputtering coefficient is then $\overline{\mathrm{S}}=1 / 2 \mathrm{~S}_{\mathrm{Be} \rightarrow \mathrm{BeO}}+1 / 2 \mathrm{~S}_{\mathrm{O} \rightarrow \mathrm{BeO}}$. For $\mathrm{B}_{4} \mathrm{C}$ the criterion is that $4 / 5 \mathrm{~S}_{\mathrm{B} \rightarrow \mathrm{B}_{4} \mathrm{C}}+1 / 5 \mathrm{~S}_{\mathrm{C} \rightarrow \mathrm{B}_{4} \mathrm{C}}<1$ and so on for ternary compounds. This condition is always met for $\mathrm{Be} O$ and $\mathrm{B}_{4} \mathrm{C}$. It is almost met everywhere for SiC. For $\mathrm{TiB}_{2}$. (not shown) it is met for $\mathrm{T}_{\mathrm{e}}<65 \mathrm{eV}$ and $\mathrm{T}_{\mathrm{e}}>1 \mathrm{keV}$ for $\mathrm{R}_{\mathrm{e}}=0$ and for $\mathrm{T}_{e}<250 \mathrm{eV}$ and $\mathrm{T}_{e}>5.5 \mathrm{keV}$ for $\mathrm{R}_{\mathrm{e}}=0.9$. For $\mathrm{TiC}$ it is met for $\mathrm{T}_{\mathrm{e}}$ $<55 \mathrm{eV}$ and $\mathrm{T}_{\mathrm{e}}>5 \mathrm{keV}$ for $\mathrm{R}_{\mathrm{e}}=0$ and for $\mathrm{T}_{\mathrm{e}}<200 \mathrm{eV}$ for $\mathrm{T}_{\mathrm{e}}<200 \mathrm{eV}$ for $R_{e}=0.9$ 


\section{EDGE-TEMPERATURE ESTIMATES}

At equilibrium a reactor-grade plasma is heated by alpha heating and this must be balanced by radiation and heat transport losses. In the bulk plasma, transport can be by conduction and convection but at the boundary heat loss occurs only by convection. The energy transported to the limiter can therefore be written as:

$$
P_{T R}=\gamma\left(K T_{e}\right) I \text {, }
$$

where $I$ is the total particle current (of all species) to the limiter and $\gamma$ is the heat transmission factor of the sheath referred to earlier. $\gamma$ is a strong function of electron re-emission because re-emitted cold electrons allow more hot electrans to reach the boundary. The transport power required to balance the alpha-heating power can be written as:

$$
P_{T R}=\left(1-f_{R}\right) P_{\alpha} \text {, }
$$

where $P_{\alpha}$ is the alpha-heating power to the plasma and $f_{R}$ is defined as the fraction of the alpha-power lost through radiation. (For this purpose, radiation is defined to include charge-exchange losses as well as the normal bremstralurg, line and recombination radiation. In general, the maximum value of $f_{R}$, that will permit equilibrium, will depend on the energy containment time of the core plasma and on transport processes in the edge region. Finally, the particle current to the limiter is given by:

$$
I=\frac{N}{\tau_{p}},
$$

where $N$ is the total number of particles in the plasma and $\tau_{p}$ is the average particle containment time (including the effects of edge recycling). Combining eqs. (1)-(3) for the edge temperature then gives the simple expression: 


$$
T_{e}=\frac{\left(1-f_{r}\right) P_{\alpha}{ }^{\tau} p}{N \gamma k} .
$$

For a typical fusion reactor design, $\mathrm{P}_{\alpha} \approx 600 \mathrm{MW}$ (corresponding to $3000 \mathrm{MW}$ thermal fusion power) and $N \approx 2 \times 10^{23}$. Using these values in eq. 4 gives an edge temperature

$$
T_{e}=\frac{1.88 \times 10^{4}\left(1-f_{R}\right) T_{p}}{\gamma} .
$$

$T_{e}$ from this formula is plotted in Figs. 5 and 6 as a function of $f_{R}$ and $\tau_{p}$ for the two limiting values of $\gamma=3.5$ corresponding to no electron re-emission and $\gamma=12$ corresponding to saturated re-emission. At the present time $\tau_{p}$ is an ambiguous parameter for present tokamaks and is quite uncertain for future devices. However, if $\tau_{p}$ is in the general range

$$
\frac{1}{2} \leq \frac{{ }^{\mathrm{T}}}{{ }_{\mathrm{T}}} \leq 3,
$$

where $\tau_{E}$ is the energy containment time, as seems to be the case at present, then for a typical value of $\tau_{E}=4 \mathrm{~s}$, edge temperatures would be well into the keV range for most values of $f_{R}$. Even for a quite low value of $\tau_{p}=0.1 \mathrm{~s}, T_{e}$ is still fairly high except for high radiated fractions. However, a low ${ }_{p}$ could well be associated with high radiation due to intense recycling and ionization in the edge region. There is apparently only a small region of the ${ }_{p}, f_{R}$ parameter space where the edge temperature would be low enough to permit $S<1$ for most of the structural metals although this region is bigger for the higher heat transmission case. 


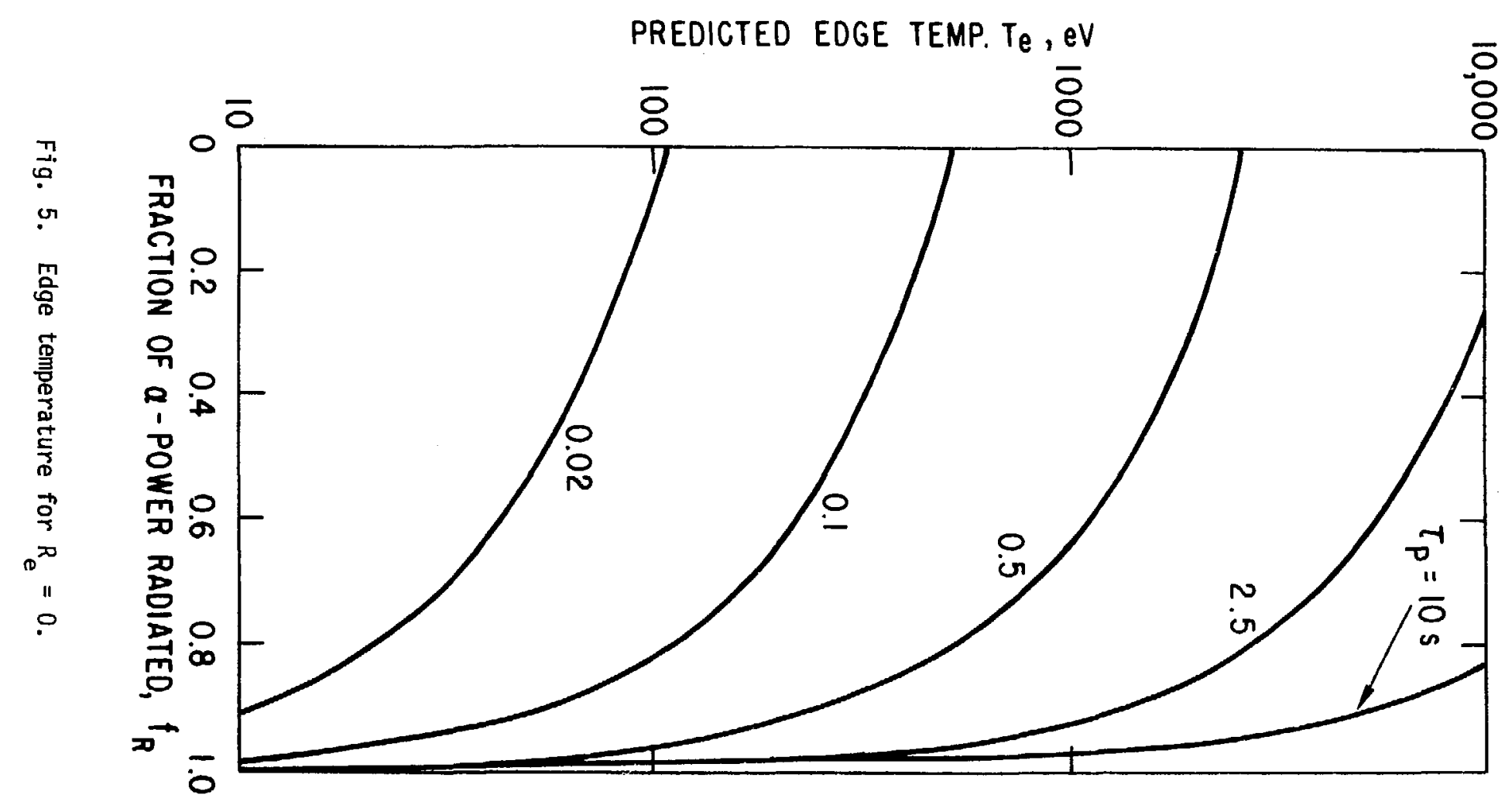




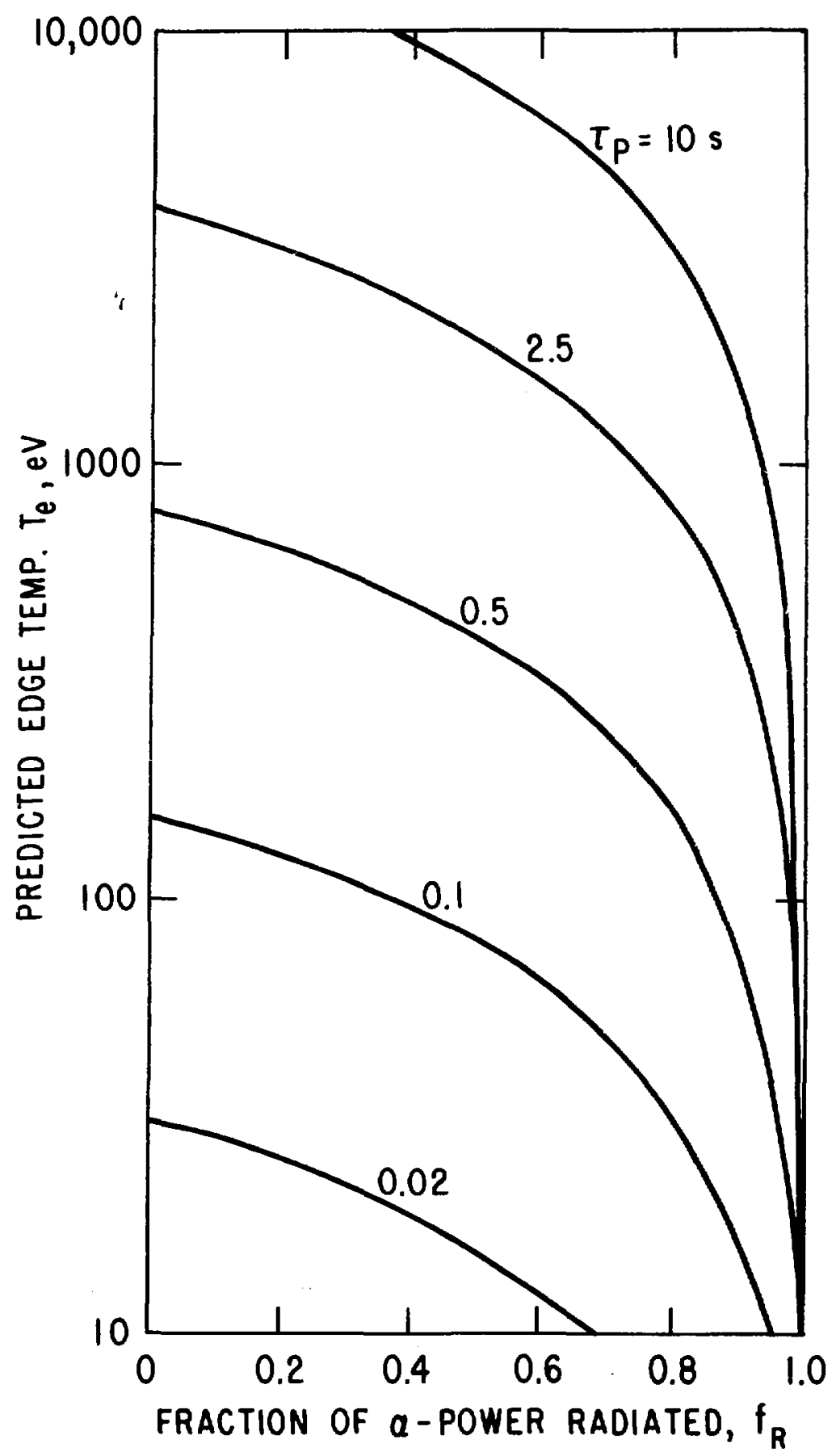

Fig. 6. Edge temperature for $R_{e}=0.9$. 


\section{CONCLUSIONS}

Calculations for self-sputtering coefficients and edge temperatures have been made for a variety of materials and for a typical fusion power reactor. From the self-sputtering standpoint, the low-Z coatings of beryllium, carbon, $\mathrm{B}_{4} \mathrm{C}$, and $\mathrm{BeO}$ are acceptable at all temperatures, $\mathrm{SiC}$ is acceptable at most temperatures, and $\mathrm{TiB}_{2}$ and $\mathrm{TiC}$ are acceptable only within certain bounds. Elements of atomic number 26 or higher appear to be acceptable only at very lowedge temperatures while aluminum may be acceptable at both low arid high-edge temperatures, but not in between. Achieving low-edge temperatures will require a combination of very short confinement times and the radiating of most of the alpha-heating power. Of course, the exact boundaries of these conclusions are subject to uncertainties in the models used. Effects which could modify these results include the use of shallow angle limiter/divertor plate configurations which could reduce sheath potentials though sputtering coefficients at a given energy could increase as well. Recent results using the kinetic theory model of ref. 7 also show a reduction in sheath potential under certain conditions of high electron re-emission, and moderate charged particle sputtering and/or $\mathrm{H}^{+}$reflection. If it were possible to control this effect, it might offer some help in relaxing the edge-temperature requirements for the higher $-Z$ materials.

\section{ACKNOWLEDGMENTS}

The author would like to acknowledge the help of Dale L. Smith in evaluating electron re-emission data and for his general comments and suggestions. 


\section{REFERENCES}

[1] C. C. Baker et al., "STARFIRE, A Commercial Tokamak Power Plant Design," to be published in Nucl. Eng. Design.

[2] D. E. Post et al., "Steady-State Radiative Cooling Rates for Low-Density High Temperature Plasmas," in Atomic Data and Nuclear Tables (Academic Press, 1978) Vol. 20, p. 397.

[3] D. L. Smith, J. Nucl. Mater. 75 (1978) 20.

[4] D. L. Smith, in Proc. Workshop on Sputtering Caused by Plasma Surface Interaction, Argonne, IL (July 1979), CONF-79-07-75 (in press).

[5] G. Fuchs and A. Nicolai, J. Nucl. Mater. $76 \& \underline{77}$ (1978) 573.

[6] P. F. HARBOUR and M. F. A. Harrison, J. Nucl. Mater. $\underline{76} \& \underline{77}$ (1978) 513.

[7] J. N. Brooks, Kinetic Study of the Sheath Region in a Fusion Reactor, Argonne National Laboratory Report, ANL/FPP/TM124 (1979).

[8] R. Kollath, as quoted in 0. Hacenberg and W. Braver, "Secondary Emission from Solids," in Advances in Electronics and Electron Physics (Academic Press, 1959) pp. 413-498.

[9] N. R. Wheten, Methods of Experimental Chemistry (Academic Press, 1963). 